\title{
Global Attractor for Damped Abstract Nonlinear Hyperbolic Systems
}

\author{
Gabriella A. Pintér \\ Center for Research in Scientific Computation \\ Department of Mathematics \\ North Carolina State University \\ Raleigh, NC 27695-8205
}

\begin{abstract}
This work is concerned with the long time dynamics of a class of abstract nonlinear second order in time systems with damping. This class of systems describes nonlinear dissipative elastic models with the nonlinear term produced by neo-Hookean type stress-strain relationships. In our earlier paper it was shown that these systems give rise to a weak dynamical system and that there exists a weak compact attractor. In the present work, using a somewhat more detailed analysis based in part on the results of H. T. Banks, D. S. Gilliam and V. I. Shubov on the existence and uniqueness of the weak solutions, we show that these systems generate a "strong" dynamical system also. More importantly, we are able to prove the existence of a compact "strong" global attractor. Finally, we make several comments concerning the regularity of this attractor, and present two examples.
\end{abstract}

\section{Introduction}

In this work we consider the following class of abstract nonlinear damped hyperbolic systems evolving in a complex separable Hilbert space $\mathcal{H}$ (actually holding in the sense of a larger space $\left.\mathcal{V}^{*}\right)$ :

$$
\begin{aligned}
& w_{t t}+\mathcal{A}_{1} w+\mathcal{A}_{2} w_{t}+\mathcal{N}^{*} g(\mathcal{N} w)=f(t) \\
& w(0)=\varphi_{0} \\
& w_{t}(0)=\varphi_{1}
\end{aligned}
$$


Here $\mathcal{A}_{1}, \mathcal{A}_{2}$ and $\mathcal{N}$ are unbounded linear operators and $g$ is a continuous nonlinear operator in $\mathcal{H}$. The precise conditions on all these operators, the forcing term $f(t)$ and on the initial data (1.2), (1.3) are given in the next section.

The global in time existence and uniqueness of the weak solution for the problem (1.1)(1.3) was shown in [BGS1, BGS2]. In this paper we continue the study of the long time behavior of the weak solutions. In our previous work $[\mathrm{P}]$ we showed that the system (1.1)(1.3) generates a weak dynamical system. The advantage of using a weak dynamical system (i.e. considering the dynamical system in the weak topology of the state space) was that bounded trajectories are precompact which is an essential property to insure the existence of an attractor. Once the existence of a weak dynamical system is shown, it is enough to establish that the system is bounded and weak point dissipative to guarantee the existence of a weak compact attractor. This idea was introduced and developed in several papers: [Ba1, Ba2, Da, Ha, Sl] (see these papers for the relevant definitions and theorems). In the present paper we establish two results. First we show that the problem (1.1)-(1.3) generates a "strong" dynamical system which is continuous in the sense that the solution depends continuously both on initial data and time. This does not follow from the earlier work since now we consider the strong topology on the state space. Our second and main result is the proof of existence of a compact global attractor. We achieve this result by showing that trajectories of the system are asymptotically compact and the dynamical system is bounded and point dissipative. The existence of the global attractor is established under the assumptions made in [BGS2] and an additional technical assumption (see A12) below). Finally, using the existence of a global Lyapunov function, we obtain that the global attractor is the unstable set of the set of its fixed points.

We briefly mention here the origin of the problem (1.1)-(1.3) and refer to [BGS2] for a more detailed discussion. In the case when the nonlinear operator $g \equiv 0$ the equation (1.1) describes a linear dissipative elastic model with $\mathcal{A}_{1}$ being the elastic operator and $\mathcal{A}_{2}$ being the dissipation operator. An extensive literature is devoted to the analysis of such models (see e.g. [BIW, CT] and references therein). The nonlinear term in equation (1.1) comes from a nonlinear constitutive law, i.e. from a neo-Hookean type stress-strain relationship. This type of constitutive laws occur in a wide range of materials which are of a great importance in modern smart material technology. Namely, the above mentioned nonlinear stress-strain relationship takes place in polymer composites such as elastomers filled with active elements. These materials are used in the development of both passive and active vibration devices. The study of systems of the type (1.1)-(1.3) is important for 
the development of computational methodologies for the identification and control of smart material composites. As was already mentioned, in this work we are primarily interested in the long-time behavior of these systems.

The present paper is organized in the following manner. In Section 2 we give a precise formulation of all necessary assumptions imposed on the problem (1.1)-(1.3). Then we state our main result: the existence of a compact global attractor. In Section 3 we prove the continuity of the dynamical system. Sections 4 and 5 are devoted to the proof of the main result - the existence of the attractor. Namely, in Section 4 we prove that our system is point-dissipative, i.e. it has an absorbing ball. In Section 5 we show that our system is asymptotically compact (see all necessary definitions below). Then by the following wellknown result, the existence of a global attractor is guaranteed [La]:

Theorem 1.1 Let $\left\{V_{t}, t \geq 0, X\right\}$ be a bounded, point-dissipative, asymptotically compact dynamical system. Then there exists a non-empty global attractor $\mathcal{A}$, which is compact and invariant. If $X$ is connected then $\mathcal{A}$ is also connected.

We collect here the definitions used in the statement of Theorem 1.1.

Definition 1.1 A dynamical system $\left\{V_{t}, t \geq 0, X\right\}$ is called bounded if for every bounded $B \subset X$ the set of positive semi-trajectories starting from $B$ (denoted by $\left.\gamma^{+}(B)\right)$ is bounded in $X$.

Definition 1.2 A dynamical system $\left\{V_{t}, t \geq 0, X\right\}$ is called point dissipative if there is a bounded set $A \subset X$ such that for any $\varepsilon>0$ and $x \in X$ there exists a $t_{0}(\varepsilon, x) \in \mathbb{R}^{+}$such that $V_{t}(x) \in \mathcal{O}_{\varepsilon}(A)$ for all $t \geq t_{0} .\left(\mathcal{O}_{\varepsilon}(A)\right.$ denotes the $\varepsilon$-neighborhood of $A$ : the union of all open balls of radius $\varepsilon$ centered at the points of $A$ ).

Definition 1.3 The dynamical system $\left\{V_{t}, t \geq 0, \mathcal{V} \times \mathcal{H}\right\}$ is called asymptotically compact if it possesses the following property: for every bounded set $B$ such that $\gamma^{+}(B)$ is bounded, each sequence of the form $\left\{V_{t_{k}}\left(x_{k}\right)\right\}_{k=1}^{\infty}$, where $x_{k} \in B$ and $t_{k} \uparrow \infty$, is precompact.

In Section 6 we show that our system has a global Lyapunov function which allows us to make some conclusions concerning the structure of the attractor. Finally, we show three examples where the existence of a global attractor is guaranteed. 


\section{Formulation of Problem}

Throughout this work we use the notations and assumptions introduced in [BGS2]. Namely, we assume that there is a sequence of separable Hilbert spaces $\mathcal{V}, \mathcal{V}_{2}, \mathcal{H}, \mathcal{V}_{2}^{*}, \mathcal{V}^{*}$ forming a Gelfand quintuple [BIW, Wl] satisfying

$$
\mathcal{V} \hookrightarrow \mathcal{V}_{2} \hookrightarrow \mathcal{H} \hookrightarrow \mathcal{V}_{2}^{*} \hookrightarrow \mathcal{V}^{*}
$$

where we assume that the embedding $\mathcal{V} \hookrightarrow \mathcal{V}_{2}$ is dense and continuous with $\|\varphi\|_{\mathcal{V}_{2}} \leq c\|\varphi\|_{\mathcal{V}}$ for $\varphi \in \mathcal{V}$ and $\mathcal{V}_{2} \hookrightarrow \mathcal{H}$ is a dense compact embedding with $\|\varphi\| \leq \tilde{c}\|\varphi\| \mathcal{V}_{2}$. We denote by $\langle,\rangle_{\mathcal{V} * \mathcal{V}}$, etc., the usual duality products [Wl]. These duality products are the extensions by continuity of the inner product in $\mathcal{H}$, denoted by $\langle$,$\rangle throughout. The norm in \mathcal{H}$ will be denoted by $\|\cdot\|$ while those in $\mathcal{V}, \mathcal{V}_{2}$ etc. will carry an appropriate subscript. The operators $\mathcal{A}_{1}$ and $\mathcal{A}_{2}$ are defined in terms of their sesquilinear forms $\sigma_{1}: \mathcal{V} \times \mathcal{V} \rightarrow \mathbb{C}$ and $\sigma_{2}: \mathcal{V}_{2} \times \mathcal{V}_{2} \rightarrow \mathbb{C}$. That is, $\mathcal{A}_{1} \in \mathcal{L}\left(\mathcal{V}, \mathcal{V}^{*}\right), \mathcal{A}_{2} \in \mathcal{L}\left(\mathcal{V}_{2}, \mathcal{V}_{2}^{*}\right)$ and $\left\langle\mathcal{A}_{1} \varphi, \psi\right\rangle_{\mathcal{V}^{*}, \mathcal{V}}=$ $\sigma_{1}(\varphi, \psi),\left\langle\mathcal{A}_{2} \varphi, \psi\right\rangle_{\mathcal{V}_{2}^{*}, \mathcal{V}_{2}}=\sigma_{2}(\varphi, \psi)$.

Let $\mathcal{L}_{T}$ denote the space of functions $w:[0, T] \rightarrow \mathcal{H}$ such that

$$
w \in C_{W}\left([0, T], \mathcal{V}_{2}\right) \cap L^{\infty}([0, T], \mathcal{V})
$$

( $W$ means weak continuity), and

$$
w_{t} \in C_{W}([0, T], \mathcal{H}) \bigcap L^{2}\left([0, T], \mathcal{V}_{2}\right)
$$

where the time derivative $w_{t}$ is understood in the sense of distributions with values in a Hilbert Space (see, e.g., [Li]). The space $\mathcal{L}_{T}$ is equipped with the norm

$$
\|w\|_{\mathcal{L}_{T}}=\operatorname{ess} \sup _{t \in[0, T]}\left(\left\|w_{t}(t)\right\|+\|w(t)\| \mathcal{V}\right)+\left(\int_{0}^{T}\left\|w_{t}(t)\right\|_{\mathcal{V}_{2}}^{2} d t\right)^{1 / 2} .
$$

Definition 2.1 We say that $w \in \mathcal{L}_{T}$ is a weak solution of the problem (1.1) - (1.3) if it satisfies the equation:

$$
\begin{aligned}
& \int_{0}^{t}\left[-\left\langle w_{\tau}(\tau), \eta_{\tau}(\tau)\right\rangle+\sigma_{1}(w(\tau), \eta(\tau))+\sigma_{2}\left(w_{\tau}(\tau), \eta(\tau)\right)+\right. \\
& +\langle g(\mathcal{N} w(\tau)), \mathcal{N} \eta(\tau)\rangle] d \tau+\left\langle w_{t}(t), \eta(t)\right\rangle= \\
& =\left\langle\varphi_{1}, \eta(0)\right\rangle+\int_{0}^{t}\langle f(\tau), \eta(\tau)\rangle_{\mathcal{V}_{2}^{*}, \mathcal{V}_{2}} d \tau
\end{aligned}
$$


for any $t \in[0, T]$ and any $\eta \in \mathcal{L}_{T}$, as well as the initial condition

$$
w(0)=\varphi_{0} .
$$

Equivalently,

$$
\left\langle w_{t t}, \eta\right\rangle_{\mathcal{V}^{*}, \mathcal{V}}+\sigma_{1}(w, \eta)+\sigma_{2}\left(w_{t}, \eta\right)+\langle g(\mathcal{N} w), \mathcal{N} \eta\rangle=\langle f, \eta\rangle_{\mathcal{V}_{2}^{*}, \mathcal{V}_{2}}
$$

is satisfied for all $\eta \in \mathcal{L}_{T}$ and for almost all $t \in[0, T]$.

In addition, we make the following assumptions (these assumptions are the same as in [BGS2] except that in A6) $f$ does not depend on $t$ and we introduce one additional assumption A12) ).

A1) The form $\sigma_{1}$ is a Hermitian sesquilinear form: for $\varphi, \psi \in \mathcal{V}$

$$
\sigma_{1}(\varphi, \psi)=\overline{\sigma_{1}(\psi, \varphi)}
$$

A2) The form $\sigma_{1}$ is $\mathcal{V}$ bounded: for $\varphi, \psi \in \mathcal{V}$

$$
\left|\sigma_{1}(\varphi, \psi)\right| \leq c_{1}\|\varphi\|_{\mathcal{V}}\|\psi\|_{\mathcal{V}} .
$$

A3) The form $\sigma_{1}$ is strictly coercive on $\mathcal{V}$ : for $\varphi \in \mathcal{V}$

$$
\operatorname{Re} \sigma_{1}(\varphi, \varphi)=\sigma_{1}(\varphi, \varphi) \geq k_{1}\|\varphi\|_{\mathcal{V}}^{2}, \quad k_{1}>0 .
$$

A4) The form $\sigma_{2}$ is bounded on $\mathcal{V}_{2}$ : for $\varphi, \psi \in \mathcal{V}_{2}$

$$
\left|\sigma_{2}(\varphi, \psi)\right| \leq c_{2}\|\varphi\|_{\nu_{2}}\|\psi\| \nu_{\nu_{2}} .
$$

A5) The real part of $\sigma_{2}$ is coercive and is symmetric on $\mathcal{V}_{2}$ :

$$
\begin{aligned}
& \operatorname{Re} \sigma_{2}(\varphi, \varphi)+\lambda_{0}\|\varphi\|^{2} \geq k_{2}\|\varphi\|_{\mathcal{V}_{2}}^{2} \quad k_{2}>0, \lambda_{0} \geq 0 \\
& \operatorname{Re} \sigma_{2}(\varphi, \psi)=\operatorname{Re} \sigma_{2}(\psi, \varphi), \text { for any } \varphi, \psi \in \mathcal{V}_{2} .
\end{aligned}
$$

A6) The forcing term $f$ is time-independent, $f \in \mathcal{V}_{2}^{*}$. 
A7) The operator $\mathcal{N}$ satisfies

$$
\mathcal{N} \in \mathcal{L}\left(\mathcal{V}_{2}, \mathcal{H}\right) \text { with }\|\mathcal{N} \varphi\| \leq \sqrt{\widetilde{k}}\|\varphi\|_{\mathcal{V}_{2}}
$$

and the range of $\mathcal{N}$ on $\mathcal{V}$ is dense in $\mathcal{H}$.

Note that (2.12) and $\mathcal{V} \hookrightarrow \mathcal{V}_{2}$ implies

$$
\mathcal{N} \in \mathcal{L}(\mathcal{V}, \mathcal{H}) \text { with }\|\mathcal{N} \varphi\| \leq \sqrt{k}\|\varphi\|_{\mathcal{V}}
$$

with $k=\tilde{c}^{2} \tilde{k}$.

A8) The nonlinear function $g: \mathcal{H} \rightarrow \mathcal{H}$ is a continuous nonlinear mapping of real gradient (or potential) type. This means that there exists a continuous Frechet-differentiable nonlinear functional $G: \mathcal{H} \rightarrow \mathbb{R}^{1}$, whose Frechet derivative $G^{\prime}(\varphi) \in \mathcal{L}\left(\mathcal{H}, \mathbb{R}^{1}\right)$ at any $\varphi \in \mathcal{H}$ can be represented in the form

$$
G^{\prime}(\varphi) \psi=\operatorname{Re}\langle g(\varphi), \psi\rangle \quad \text { for any } \psi \in \mathcal{H}
$$

We also require that there are constants $C_{1}, C_{2}, C_{3}$ and $\varepsilon>0$ such that

$$
-\frac{1}{2} k^{-1}\left(k_{1}-\varepsilon\right)\|\varphi\|^{2}-C_{1} \leq G(\varphi) \leq C_{2}\|\varphi\|^{2}+C_{3}
$$

where $k$ is from (2.13) and $k_{1}$ from (2.8).

A9) The nonlinear function $g$ also satisfies

$$
\|g(\varphi)\| \leq \widetilde{C}_{1}\|\varphi\|+\widetilde{C}_{2}, \quad \varphi \in \mathcal{H}
$$

for some constants $\tilde{C}_{1}, \tilde{C}_{2}$.

An additional condition is necessary for uniqueness of solutions.

A10) For any $\varphi \in \mathcal{H}$ the Frechet derivative of $g$ exists and satisfies

$$
g^{\prime}(\varphi) \in \mathcal{L}(\mathcal{H}, \mathcal{H}) \text { with }\left\|g^{\prime}(\varphi)\right\|_{\mathcal{L}(\mathcal{H}, \mathcal{H})} \leq \widetilde{C}_{3}
$$

A11) We assume that for any $u, v \in \mathcal{L}_{T}$, the following inequality is satisfied for any $t \in[0, T]$ :

$$
\begin{aligned}
& \int_{0}^{t}\{\operatorname{Re}\langle g(\mathcal{N} u(\tau))-g(\mathcal{N} v(\tau)), \mathcal{N} u(\tau)-\mathcal{N} v(\tau)\rangle \\
& \left.+k_{1} k^{-1}\|\mathcal{N} u(\tau)-\mathcal{N} v(\tau)\|^{2}\right\} d t \\
& +a\left(\left(\int_{0}^{t}\|u(\tau)-v(\tau)\|^{2} d t\right)^{1 / 2}\right) \geq 0
\end{aligned}
$$

where $a(\xi) \geq 0$ is a continuous function in $\xi \geq 0$ such that 
i) $a(0)=0$,

ii) there exists a first derivative such that $a^{\prime}(0)=0$.

Note that (2.18) is satisfied if, for example,

$$
\operatorname{Re}\langle g(\varphi)-g(\psi), \varphi-\psi\rangle+k_{1} k^{-1}\|\varphi-\psi\|^{2} \geq 0
$$

for any $\varphi, \psi \in \mathcal{H}$, where $k$ and $k_{1}$ are the constants in (2.13) and (2.8).

E.g. if $\mathcal{H}=L^{2}(\Omega), \Omega \subset \mathbb{R}^{m}$, so that $g: \mathbb{R} \rightarrow \mathbb{R}$, then a sufficient condition for (2.19) is that $g^{\prime}(\xi) \geq-l_{1}$ for some $l_{1}>0$.

A12) The embedding $\mathcal{V} \hookrightarrow \mathcal{V}_{2}$ is compact.

Some of the considerations below do not use all the assumptions A1)-A12), but only a part of them. In particular, A12) is necessary only to show the asymptotic compactness of our system and, therefore, the existence of the attractor.

We mention that the main difficulty in dealing with the problem (1.1)-(1.3) comes from the fact that the nonlinearity is very strong. The proof of the global in time existence and uniqueness for this problem [BGS2] involves the Minty-Browder monotonicity method [LSU, B, M, Li]. Due to the above mentioned difficulty our result does not follow from well-known results on attractors for dissipative hyperbolic equations [La, $\mathrm{H}, \mathrm{Te}$ ].

Now we are in a position to state precisely the main result of this paper - the existence of a global attractor. Our other results - the continuity of the dynamical system and the regularity of the attractor are formulated in Section 3 and 6 respectively.

Theorem 2.1 Under conditions A1)-A12) the system (1.1)-(1.3) has a non-empty global attractor $\mathcal{A} \subset \mathcal{V} \times \mathcal{H}$, which is compact, connected and invariant under the flow.

The proof of Theorem 2.1 is contained in a series of results in Section 3 through Section 5.

\section{The Dynamical System}

At this point we recall the following two earlier results, one from [BIW] and the other from [BGS2].

In $[\mathrm{BIW}$ the linear system

$$
\begin{aligned}
& y_{t t}+\mathcal{A}_{1} y+\mathcal{A}_{2} y_{t}=h(t) \\
& y(0)=\varphi_{0} \\
& y_{t}(0)=\varphi_{1}
\end{aligned}
$$


is investigated and the following result is obtained.

Theorem 3.1 Suppose that $\sigma_{1}$ and $\sigma_{2}$ satisfy the conditions $\left.\left.A 1\right)-A 5\right), h \in L^{2}\left([0, T], \mathcal{V}_{2}^{*}\right)$ and $\varphi_{0} \in \mathcal{V}, \varphi_{1} \in \mathcal{H}$. Then (3.1)-(3.3) has a unique weak solution y satisfying

$$
\begin{aligned}
& \left\langle y_{t t}, \varphi\right\rangle_{\mathcal{V} *, \mathcal{V}}+\sigma_{1}(y, \varphi)+\sigma_{2}\left(y_{t}, \varphi\right)=\langle h, \varphi\rangle_{\mathcal{V}_{2}^{*}, \mathcal{V}_{2}} \\
& y(0)=\varphi_{0} \\
& y_{t}(0)=\varphi_{1}
\end{aligned}
$$

for all $\varphi \in \mathcal{V}$ and for almost all $t \in[0, T]$ with

$$
y \in C([0, T], \mathcal{V}), y_{t} \in C([0, T], \mathcal{H}) \cap L^{2}\left([0, T], \mathcal{V}_{2}\right)
$$

and the map $\left(\varphi_{0}, \varphi_{1}, h\right) \rightarrow y$ is continuous from $\mathcal{V} \times \mathcal{H} \times L^{2}\left([0, T], \mathcal{V}_{2}^{*}\right)$ to

$$
\mathcal{Z} \equiv\left\{z \in C((0, T), \mathcal{V}) \mid z_{t} \in C([0, T], \mathcal{H}) \cap L^{2}\left((0, T), \mathcal{V}_{2}\right)\right\}
$$

where the norm in $\mathcal{Z}$ is given by

$$
\|z\|_{\mathcal{Z}}=\sup _{t \in[0, T]}\left\{\|z(t)\|_{\mathcal{V}}+\left\|z_{t}(t)\right\|\right\}+\left(\int_{0}^{T}\left\|z_{s}(s)\right\|_{\mathcal{V}_{2}}^{2} d s\right)^{1 / 2} .
$$

In $[\mathrm{BGS2}]$ it is shown that

Theorem 3.2 Under conditions A1)-A11) the system (1.1)-(1.3) has a unique weak solution $w \in \mathcal{L}_{T}$ for every initial condition $\left(\begin{array}{c}\varphi_{0} \\ \varphi_{1}\end{array}\right) \in \mathcal{V} \times \mathcal{H}$. The weak solution satisfies

$$
\left\langle w_{t t}, \eta\right\rangle_{\mathcal{V} * \mathcal{V}}+\sigma_{1}(w, \eta)+\sigma_{2}\left(w_{t}, \eta\right)+\langle g(\mathcal{N} w), \mathcal{N} \eta\rangle=\langle f, \eta\rangle_{\mathcal{V}_{2}^{*}, \mathcal{V}_{2}}
$$

for all $\eta \in \mathcal{L}_{T}$ and for almost all $t \in[0, T]$.

Since the existence of global weak solutions of (1.1)-(1.3) is guaranteed by the above Theorem 3.2 we can define the solution operator $V_{t}: \mathcal{V} \times \mathcal{H} \rightarrow \mathcal{V} \times \mathcal{H}$ by

$$
V_{t}\left(\begin{array}{c}
\varphi_{0} \\
\varphi_{1}
\end{array}\right)=\left(\begin{array}{c}
w(t) \\
w_{t}(t)
\end{array}\right)
$$

where $w$ is the weak solution of (1.1) corresponding to the initial condition $\left(\begin{array}{l}\varphi_{0} \\ \varphi_{1}\end{array}\right)$. The main result of this section is the following: 
Theorem 3.3 If conditions A1)-A11) are satisfied, then $\left\{V_{t}, t \geq 0, \mathcal{V} \times \mathcal{H}\right\}$ is a continuous dynamical system in the sense that $\left\{V_{t}, t \geq 0\right\}$ is a semigroup on $\mathcal{V} \times \mathcal{H}$ and the mapping

$$
\begin{gathered}
V: \mathbb{R}^{+} \times \mathcal{V} \times \mathcal{H} \rightarrow \mathcal{V} \times \mathcal{H}: \\
\left(t, \varphi_{0}, \varphi_{1}\right) \rightarrow V_{t}\left(\begin{array}{c}
\varphi_{0} \\
\varphi_{1}
\end{array}\right)
\end{gathered}
$$

is continuous.

Proof: It is clear that $\left\{V_{t}, t \geq 0, \mathcal{V} \times \mathcal{H}\right\}$ is a semigroup since (by A6) $f$ does not depend on $t$. The continuity statement follows from the next two lemmas.

Lemma 3.1 The weak solution depends continuously on initial conditions, i.e. for a fixed $t_{0} \geq 0, V_{t_{0}}$ is a continuous operator.

Proof of Lemma 3.1: Let $w$ and $\tilde{w}$ be weak solutions of (2.1) with initial conditions $\varphi_{0}, \varphi_{1}$ and $\tilde{\varphi}_{0}, \tilde{\varphi}_{1}$, respectively. Let $u=w-\tilde{w}, \psi_{0}=\varphi_{0}-\tilde{\varphi}_{0}$ and $\psi_{1}=\varphi_{1}-\tilde{\varphi}_{1}$. Then from (3.8) we have

$$
\left\langle u_{t t}, \eta\right\rangle_{\mathcal{V}^{*}, \mathcal{V}}+\sigma_{1}(u, \eta)+\sigma_{2}\left(u_{t}, \eta\right)+\langle g(\mathcal{N} w), \mathcal{N} \eta\rangle-\langle g(\mathcal{N} \tilde{w}), \mathcal{N} \eta\rangle=0
$$

for all $\eta \in \mathcal{L}_{T}$ and for almost all $t \in[0, T]$. Let $\Delta g=g(\mathcal{N} w)-g(\mathcal{N} \tilde{w})$. Choosing $\eta=u_{t}$ we formally $\left(u_{t} \notin \mathcal{L}_{T}\right)$ get:

$$
\frac{d}{d t}\left\{\frac{1}{2}\left\|u_{t}\right\|^{2}+\frac{1}{2} \sigma_{1}(u, u)\right\}+\sigma_{2}\left(u_{t}, u_{t}\right)+\left\langle\Delta g, \mathcal{N} u_{t}\right\rangle=0 .
$$

Taking the real part and integrating from 0 to $t$ we obtain:

$$
\begin{gathered}
\left\|u_{t}(t)\right\|^{2}+\sigma_{1}(u(t), u(t))+2 \operatorname{Re} \int_{0}^{t} \sigma_{2}\left(u_{\tau}(\tau), u_{\tau}(\tau)\right) d \tau= \\
-2 \operatorname{Re} \int_{0}^{t}\left\langle\Delta g, \mathcal{N} u_{\tau}(\tau)\right\rangle d \tau+\left\|u_{t}(0)\right\|^{2}+\sigma_{1}(u(0), u(0)) .
\end{gathered}
$$

Using the following estimate from [BGS2]:

$$
\left|\int_{0}^{t}\left\langle\Delta g, \mathcal{N} u_{\tau}\right\rangle\right| \leq \widetilde{C_{3}} \frac{1}{4 \delta} k \int_{0}^{t}\|u(\tau)\|_{\mathcal{V}}^{2} d \tau+\widetilde{C_{3}} \delta \tilde{k} \int_{0}^{t}\left\|u_{\tau}(\tau)\right\|_{\mathcal{V}_{2}}^{2} d \tau,
$$

(where $\delta>0$ arbitrary) and A3), A5) we have:

$$
\begin{aligned}
& \left\|u_{t}(t)\right\|^{2}+k_{1}\|u(t)\|_{\mathcal{V}}^{2}+\left(2 k_{2}-2 \widetilde{C_{3}} \delta \tilde{k}\right) \int_{0}^{t}\left\|u_{\tau}(\tau)\right\|_{\mathcal{V}_{2}}^{2} d \tau \leq 2 \lambda_{0} \int_{0}^{t}\left\|u_{\tau}(\tau)\right\|^{2} d \tau \\
& +\frac{\widetilde{C_{3}} k}{2 \delta} \int_{0}^{t}\|u(\tau)\|_{\mathcal{V}}^{2} d \tau+\left\|\psi_{1}\right\|^{2}+c_{1}\left\|\psi_{0}\right\|_{\mathcal{V}}^{2} .
\end{aligned}
$$


Let $\delta=\delta_{0}$ where we choose $\delta_{0}$ such that $k_{2}-2 \widetilde{C_{3}} \tilde{k} \delta>0\left(\right.$ such a $\delta_{0}$ exists since $\left.k_{2}, \widetilde{C_{3}}, \tilde{k}>0\right)$. Then from (3.12) we have:

$$
\left\|u_{t}\right\|^{2}+\|u(t)\|_{\mathcal{V}}^{2} \leq d_{1} \int_{0}^{t}\left(\left\|u_{\tau}(\tau)\right\|^{2}+\|u(\tau)\|_{\mathcal{V}}^{2}\right) d \tau+d_{2}\left(\left\|\psi_{1}\right\|^{2}+\left\|\psi_{0}\right\|_{\mathcal{V}}^{2}\right)
$$

where $d_{1}=\frac{\max \left(2 \lambda_{0}, \widetilde{C_{3} k}\right)}{\min \left(1, k_{1}\right)}$ and $d_{2}=\frac{\max \left(1, c_{1}\right)}{\min \left(1, k_{1}\right)}$.

By Gronwall's inequality:

$$
\left\|u_{t}(t)\right\|^{2}+\|u(t)\|_{\mathcal{V}}^{2} \leq\left(d_{2}\left\|\psi_{1}\right\|^{2}+\left\|\psi_{0}\right\|_{\mathcal{V}}^{2}\right) e^{d_{1} t}
$$

For $t=t_{0}$ we finally obtain:

$$
\left\|u_{t}\left(t_{0}\right)\right\|^{2}+\left\|u\left(t_{0}\right)\right\|_{\mathcal{V}}^{2} \leq d_{2}\left(\left\|\psi_{1}\right\|^{2}+\left\|\psi_{0}\right\|_{\mathcal{V}}^{2}\right) e^{d_{1} t_{0}}
$$

Since the last factor is clearly bounded for a fixed $t_{0} \in[0, T]$, we have the required continuity result for a fixed $t_{0}$. The fact that this result is actually valid for the weak solution can be justified by considering the Galerkin approximates $w_{t}^{N}(t), w^{N}(t)$ [BGS2], for which (3.15) is certainly true, and then utilizing the convergences $w^{N}(t) \rightarrow w(t)$ weakly in $\mathcal{V}$ and $w_{t}^{N}(t) \rightarrow$ $w_{t}(t)$ weakly in $\mathcal{H}, \varphi_{0}^{N} \rightarrow \varphi_{0}$ strongly in $\mathcal{V}, \varphi_{1}^{N} \rightarrow \varphi_{1}$ strongly in $\mathcal{H}$, and using the weak lower semicontinuity of norms in Hilbert space.

In the following lemma we prove that $V_{t}$ is continuous with respect to $t$.

Lemma 3.2 For a fixed initial condition $\left(\begin{array}{c}\varphi_{0} \\ \varphi_{1}\end{array}\right) \in \mathcal{V} \times \mathcal{H}, V_{t}\left(\begin{array}{c}\varphi_{0} \\ \varphi_{1}\end{array}\right)$ is continuous in $t$

Proof of Lemma 3.2: From Theorem 3.2 we only know that

$$
w \in C_{W}([0, T], \mathcal{V}) \text { and } w_{t} \in C_{W}([0, T], \mathcal{H})
$$

To show strong continuity we are going to use Theorem 3.1 recalled from [BIW] at the beginning of this section. We consider the following two systems:

$$
\begin{aligned}
& u_{t t}+\mathcal{A}_{1} u+\mathcal{A}_{2} u_{t}=f-\mathcal{N}^{*} g(\mathcal{N} w) \\
& u(0)=u_{t}(0)=0
\end{aligned}
$$


and

$$
\begin{aligned}
& v_{t t}+\mathcal{A}_{1} v+\mathcal{A}_{2} v_{t}=0 \\
& v(0)=\varphi_{0} \\
& v_{t}(0)=\varphi_{1}
\end{aligned}
$$

where $w$ is the weak solution of (1.1)-(1.3). We have the following estimate for the right hand side of (3.17):

$$
\begin{aligned}
& \left\|f-\mathcal{N}^{*} g(\mathcal{N} w)\right\|_{L^{2}\left([0, T], \mathcal{V}_{2}^{*}\right)}^{2}=\int_{0}^{T}\left\|f-\mathcal{N}^{*} g(\mathcal{N} w)\right\|_{\mathcal{V}_{2}^{*}}^{2} d \tau \\
& \leq 2 \int_{0}^{T}\left(\|f\|_{\mathcal{V}_{2}^{*}}^{2}+\| \mathcal{N}^{*}\left(g(\mathcal{N} w) \|_{\mathcal{V}_{2}^{*}}^{2}\right) d \tau\right. \\
& \leq 2 T\|f\|_{\mathcal{V}_{2}^{*}}^{2}+2 \tilde{k} \int_{0}^{T}\|g(\mathcal{N} w)\|^{2} d \tau \leq 2 T\|f\|_{\mathcal{V}_{2}^{*}}^{2} \\
& +2 \tilde{k} \int_{0}^{T}\left(\tilde{C}_{1}^{2}\|\mathcal{N} w\|^{2}+2 \tilde{C}_{1} \tilde{C}_{2}\|\mathcal{N} w\|+\tilde{C}_{2}^{2}\right) d \tau \\
& \leq 2 T\|f\|_{\mathcal{V}_{2}^{*}}^{2}+2 \tilde{C}_{1}^{2} \tilde{k}^{2} \int_{0}^{T}\|w(\tau)\|_{\mathcal{V}_{2}}^{2} d \tau \\
& +4 \tilde{k} \tilde{C}_{1} \tilde{C}_{2} \sqrt{k} \int_{0}^{T}\|w(\tau)\| \mathcal{V} d \tau+2 \tilde{k} T \tilde{C}_{2}^{2},
\end{aligned}
$$

which is finite since $w \in L^{\infty}([0, T], \mathcal{V}) \cap L^{2}\left([0, T], \mathcal{V}_{2}\right)$ (see [BGS2]). (Here $\tilde{k}$ is the constant from $(2.12)$ so that $\left\|\mathcal{N}^{*} \varphi\right\|_{\mathcal{V}_{2}^{*}} \leq \sqrt{\tilde{k}}\|\varphi\|$, since $\left.\|\mathcal{N}\|=\left\|\mathcal{N}^{*}\right\|.\right)$ It follows that

$$
h=f-\mathcal{N}^{*} g(\mathcal{N} w) \in L^{2}\left([0, T], \mathcal{V}_{2}^{*}\right),
$$

so by Theorem 3.1 the unique weak solutions $u$ and $v$ of (3.16)-(3.17) and (3.18)-(3.20) respectively, satisfy $u, v \in C([0, T], \mathcal{V}), u_{t}, v_{t} \in C([0, T], \mathcal{H})$. However, $u+v-w$ is a weak solution of (3.1)-(3.3) with $h=\varphi_{0}=\varphi_{1}=0$, so by uniqueness $w=u+v$. It follows that $w \in C([0, T], \mathcal{V})$ and $w_{t} \in C([0, T], \mathcal{H})$, so we have continuity in $t$. The proof of Theorem 3.3 is complete.

\section{Point-dissipativity}

In this section we prove the following theorem: 
Theorem 4.1 Under conditions A1)-A11) the dynamical system $\left\{V_{t}, t \geq 0, \mathcal{V} \times \mathcal{H}\right\}$ is bounded and point-dissipative.

Proof: We again proceed formally. Choosing $\eta=w_{t}$ in (3.8) and taking the real part we obtain:

$$
\frac{d}{d t}\left\{\frac{1}{2}\left\|w_{t}\right\|^{2}+\frac{1}{2} \sigma_{1}(w, w)+G(\mathcal{N} w)\right\}+\operatorname{Re} \sigma_{2}\left(w_{t}, w_{t}\right)=\operatorname{Re}\left\langle f, w_{t}\right\rangle_{\mathcal{V}_{2}^{*}, \mathcal{V}_{2}}
$$

where we have used the fact that due to (2.14) we have

$$
\frac{d}{d t} G(\mathcal{N} w)=\operatorname{Re}\left\langle g(\mathcal{N} w), \mathcal{N} w_{t}\right\rangle
$$

Using A5) and multiplying by 2 we get:

$$
\frac{d}{d t}\left\{\left\|w_{t}\right\|^{2}+\sigma_{1}(w, w)+2 G(\mathcal{N} w)\right\}+2 k_{2}\left\|w_{t}\right\|_{\mathcal{V}_{2}}^{2}-2 \lambda_{0}\left\|w_{t}\right\|^{2} \leq 2 \delta\left\|w_{t}\right\|_{\mathcal{V}_{2}}^{2}+\frac{1}{2 \delta}\|f\|_{\mathcal{V}_{2}^{*}}^{2}
$$

Let $l=\frac{2\left(k_{2}-\delta\right)}{\tilde{c}}$, where we choose $\delta$ such that $k_{2}-\delta>0$. Then by (2.1) we have that $2\left(k_{2}-\delta\right)\left\|w_{t}\right\|_{\mathcal{V}_{2}}^{2} \geq \frac{2\left(k_{2}-\delta\right)}{\tilde{c}}\left\|w_{t}\right\|^{2}$. Using this inequality in (4.2), multiplying by $e^{l t}$ and then integrating from 0 to $t$ we obtain:

$$
\begin{aligned}
& e^{l t}\left\|w_{t}(t)\right\|^{2}-\left\|w_{t}(0)\right\|^{2}+\int_{0}^{t} e^{l s} \frac{d}{d s} \sigma_{1}(w(s), w(s)) d s+\int_{0}^{t} 2 e^{l s} \frac{d}{d s}(G \mathcal{N} w(s)) d s \\
& \leq \frac{1}{2 \delta} \int_{0}^{t} e^{l s}\|f\|_{\mathcal{V}_{2}^{*}}^{2} d s+2 \lambda_{0} \int_{0}^{t} e^{l s}\left\|w_{s}(s)\right\|^{2} d s
\end{aligned}
$$

An integration by parts leads to:

$$
\begin{aligned}
& e^{l t}\left\|w_{t}(t)\right\|^{2}-\left\|\varphi_{1}\right\|^{2}+e^{l t} \sigma_{1}(w(t), w(t))-\sigma_{1}(w(0), w(0)) \\
& -\int_{0}^{t} l e^{l s} \sigma_{1}(w(s), w(s)) d s+2 e^{l t} G(\mathcal{N} w(t))-2 G(\mathcal{N} w(0))- \\
& \int_{0}^{t} 2 l e^{l s} G(\mathcal{N} w(s)) d s \leq \frac{1}{2 \delta} \int_{0}^{t} e^{l s}\|f\|_{\mathcal{V}_{2}^{*}}^{2} d s+2 \lambda_{0} \int_{0}^{t} e^{l s}\left\|w_{s}(s)\right\|^{2} d s .
\end{aligned}
$$

This gives (using A3), A2) )

$$
\begin{aligned}
& \left\|w_{t}(t)\right\|^{2}+k_{1}\|w(t)\|_{\mathcal{V}}^{2}+2 G(\mathcal{N} w(t)) \leq \\
& e^{-l t}\left(\left\|\varphi_{1}\right\|^{2}+c_{1}\left\|\varphi_{0}\right\|_{\mathcal{V}}^{2}+2 G(\mathcal{N} w(0))\right) \\
& +l \int_{0}^{t} e^{l(s-t)} \sigma_{1}(w(s), w(s)) d s+2 l \int_{0}^{t} e^{l(s-t)} G(\mathcal{N} w(s)) d s \\
& +\frac{1}{2 \delta} \int_{0}^{t} e^{l(s-t)}\|f\|_{\mathcal{V}_{2}^{*}}^{2} d s+2 \lambda_{0} \int_{0}^{t} e^{l(s-t)}\left\|w_{s}(s)\right\|^{2} d s .
\end{aligned}
$$


By A8), A7), A3) we obtain:

$$
\begin{aligned}
& \left\|w_{t}(t)\right\|^{2}+k_{1}\|w(t)\|_{\mathcal{V}}^{2}+2\left[-\frac{1}{2} k^{-1}\left(k_{1}-\varepsilon\right) k\|w(t)\|_{\mathcal{V}}^{2}-C_{1}\right] \leq \\
& e^{-l t}\left[\left\|\varphi_{1}\right\|^{2}+c_{1}\left\|\varphi_{0}\right\|_{\mathcal{V}}^{2}+2 C_{2} k\left\|\varphi_{0}\right\|_{\mathcal{V}}^{2}+2 C_{3}\right]+l c_{1} \int_{0}^{t} e^{l(s-t)}\|w(s)\|_{\mathcal{V}}^{2} d s \\
& +2 l \int_{0}^{t} e^{l(s-t)}\left(C_{2} k\|w(s)\|_{\mathcal{V}}^{2}+C_{3}\right) d s \\
& +\frac{1}{2 \delta} \int_{0}^{t} e^{l(s-t)}\|f\|_{\mathcal{V}_{2}^{*}}^{2} d s+2 \lambda_{0} \int_{0}^{t} e^{l(s-t)}\left\|w_{s}(s)\right\|^{2} d s .
\end{aligned}
$$

This gives

$$
\begin{aligned}
& \left\|w_{t}(t)\right\|^{2}+\varepsilon\|w(t)\|_{\mathcal{V}}^{2} \leq e^{-l t} K+\left(2 l C_{3}+\frac{\|f\|_{\mathcal{V}_{2}^{*}}^{2}}{2 \delta}\right) \int_{0}^{t} e^{l(s-t)} d s \\
& +2 C_{1}+L \int_{0}^{t} e^{l(s-t)}\left(\|w(s)\|_{\mathcal{V}}^{2}+\left\|w_{s}(s)\right\|^{2}\right) d s
\end{aligned}
$$

where $K=\left[\left\|\varphi_{1}\right\|^{2}+c_{1}\left\|\varphi_{0}\right\|_{\mathcal{V}}^{2}+2 C_{2} k\left\|\varphi_{0}\right\|_{\mathcal{V}}^{2}+2 C_{3}\right]$, and $L=\max \left(l c_{1}+2 l C_{2} k, 2 \lambda_{0}\right)$.

Let $\tilde{\varepsilon}=\min (1, \varepsilon), L_{1}=L \tilde{\varepsilon}^{-1}, K_{1}=K \tilde{\varepsilon}^{-1}, K_{2}=\left(2 l C_{3}+\frac{\|f\|_{\mathcal{V}_{2}^{*}}^{2}}{2 \delta}\right) \tilde{\varepsilon}^{-1} l$ and $K_{3}=2 C_{1} \tilde{\varepsilon}^{-1}$. With these, using Gronwall's inequality, we get:

$$
\begin{aligned}
& \left\|w_{t}(t)\right\|^{2}+\|w(t)\|_{\mathcal{V}}^{2} \leq K_{1} e^{-l t}+K_{2}\left(1-e^{-l t}\right)+K_{3} \\
& +L_{1} \int_{0}^{t} e^{l(s-t)}\left[K_{1} e^{-l s}+K_{2}\left(1-e^{-l s}\right)+K_{3}\right] e^{\int_{s}^{t} L_{1} e^{l(\theta-t)} d \theta} d s \\
& \leq K_{1} e^{-l t}+K_{2}\left(1-e^{-l t}\right)+K_{3} \\
& +L_{1} \int_{0}^{t}\left(e^{-l t}\left(K_{1}-K_{2}\right)+\left(K_{2}+K_{3}\right) e^{l(s-t)}\right) e^{\frac{L_{1}}{l}} d s \\
& \leq K_{1} e^{-l t}+K_{3}+K_{2}\left(1-e^{-l t}\right)+L_{1} e^{\frac{L_{1}}{l}}\left|K_{1}-K_{2}\right| t e^{-l t}+L_{1}\left(\frac{K_{2}+K_{3}}{l}\right) e^{\frac{L_{1}}{l}}
\end{aligned}
$$

Here only $K_{1}=\tilde{\varepsilon}^{-1}\left(\left\|\varphi_{1}\right\|^{2}+c_{1}\left\|\varphi_{0}\right\|_{\mathcal{V}}^{2}+2 C_{2} k\left\|\varphi_{0}\right\|_{\mathcal{V}}^{2}+2 C_{3}\right)$ depends on the initial conditions $\left(K_{2}, K_{3}, L_{1}\right.$ and $l$ are independent of them), so given any $\Phi=\left(\begin{array}{c}\varphi_{0} \\ \varphi_{1}\end{array}\right) \in \mathcal{V} \times \mathcal{H}$ and $\varepsilon>0$ there exists a $t_{0}>0$ such that for $t>t_{0}$, we have $\left|e^{-l t} K_{1}\right|<\frac{\varepsilon}{2}$ and $\left|K_{1}-K_{2}\right| t e^{-l t}<\frac{\varepsilon}{2}$, so

$$
\left\|w_{t}(t)\right\|^{2}+\|w(t)\|_{\mathcal{V}}^{2} \leq \varepsilon+K_{3}+K_{2}+L_{1}\left(\frac{K_{2}+K_{3}}{l}\right) e^{\frac{L_{1}}{l}} .
$$

This means that the dynamical system is point-dissipative, i.e. the ball of radius

$$
R=\sqrt{\left(K_{3}+K_{2}\right)+L_{1}\left(\frac{K_{2}+K_{3}}{l}\right) e^{\frac{L_{1}}{l}}}
$$


in $\mathcal{V} \times \mathcal{H}$ attracts every element of $\mathcal{V} \times \mathcal{H}$. The estimate (4.8) also shows that the dynamical system is bounded, i.e. if $B \subset \mathcal{V} \times \mathcal{H}$ is bounded, then $V_{t}(B)=\cup_{\Phi \in B} V_{t}(\Phi)$ is bounded. (Again to justify that these estimates are correct for the weak solution we have to consider the Galerkin approximates as in Section 3.)

\section{The Global Attractor}

Now we know that the dynamical system $\left\{V_{t}, t \geq 0, \mathcal{V} \times \mathcal{H}\right\}$ is bounded and point-dissipative, so from Theorem 1.1 the last thing we need to show is the asymptotic compactness in order to complete the proof of our main result Theorem 2.1. To achieve this, we will use the additional assumption A12) (i.e. the embedding $\mathcal{V} \hookrightarrow \mathcal{V}_{2}$ is compact). Let us recall the following theorem from $[\mathrm{La}]$ :

Theorem 5.1 Suppose that the dynamical system $\left\{V_{t}, t \geq 0, X\right\}$ can be decomposed to a sum $W_{t}+U_{t}$, where $\left\{W_{t}, t \geq 0, X\right\}$ is a family of operators such that for any bounded set $B \subset X$

$$
\left\|W_{t}(B)\right\|_{X} \leq m_{1}(t) m_{2}\left(\|B\|_{X}\right)
$$

where $m_{k}: \mathbb{R}^{+} \rightarrow \mathbb{R}^{+}$are continuous for $k=1,2$ and $m_{1}(t) \rightarrow 0$ as $t \rightarrow \infty,\|B\|_{X}:=$ $\sup _{x \in B}\|x\|_{X}$. If the operators $U_{t}$ are such that the set $U_{t}(B)$ is precompact for each bounded set $B \subset X$ and $t>0$, then $\left\{V_{t}, t \geq 0, X\right\}$ is asymptotically compact.

Proof of asymptotic compactness of $V_{t}$ : Consider systems (3.16)-(3.17) and (3.18)(3.20) again. Let $W_{t}$ and $U_{t}$ be the solution operator of (3.16)-(3.17) and (3.18)-(3.20), respectively. First, we show that the set $U_{t}(B)$ is precompact for any bounded $B \subset \mathcal{V} \times \mathcal{H}$ (we use an idea similar to one found in $[\mathrm{Fa}]$ ).

Lemma 5.1 For any bounded $B \subset \mathcal{V} \times \mathcal{H}$ and for any $T>0$ the set

$$
U_{T}(B)=\left\{\left(u(T), u_{t}(T)\right) \mid\left(u, u_{t}\right) \text { is a weak solution of (3.16)-(3.17) for }\left(\begin{array}{l}
\varphi_{0} \\
\varphi_{1}
\end{array}\right) \in B\right\}
$$

is precompact in $\mathcal{V} \times \mathcal{H}$.

Proof of Lemma 5.1: Let us fix a bounded $B \subset \mathcal{V} \times \mathcal{H}$ and $T>0$. Let $\Psi: L \rightarrow \mathcal{V} \times \mathcal{H}$, where

$$
L \equiv\left\{w \in C\left([0, T], \mathcal{V}_{2}\right),: w \text { the weak solution of }(1.1) \text { with }\left(\begin{array}{c}
w(0) \\
w_{t}(0)
\end{array}\right)=\left(\begin{array}{c}
\varphi_{0} \\
\varphi_{1}
\end{array}\right) \in B\right\}
$$


be defined by: $\Psi(w)=\left(u(T), u_{t}(T)\right)$, where $u$ is the unique weak solution of (3.16)-(3.17) with $w$ on the right in (3.16).

First we show that $\Psi$ is continuous.

Let $\left(u, u_{t}\right)$ be the weak solution of (3.16)-(3.17) corresponding to $\left(w, w_{t}\right)$ which is the weak solution of $(1.1)$ with initial conditions $\left(\begin{array}{l}\varphi_{0} \\ \varphi_{1}\end{array}\right) \in B$. We need to show that for every $\varepsilon>0$, there exists a $\delta>0$ such that if

$$
\|w-\tilde{w}\|_{C\left([0, T], \mathcal{V}_{2}\right)}<\delta, \tilde{w} \in L \text { then }\left\|\left(\begin{array}{c}
u(T)-\tilde{u}(T) \\
u_{t}(T)-\tilde{u}_{t}(T)
\end{array}\right)\right\|_{\mathcal{V} \times \mathcal{H}}<\varepsilon,
$$

where $\left(\begin{array}{c}\tilde{u} \\ \tilde{u}_{t}\end{array}\right)$ is the weak solution of (3.16)-(3.17) corresponding to $\tilde{w} \in L$. If $u$ and $\tilde{u}$ are as above then $\left(z, z_{t}\right)=\left(u-\tilde{u}, u_{t}-\tilde{u}_{t}\right)$ is a weak solution of

$$
\begin{aligned}
& z_{t t}+\mathcal{A}_{1} z+\mathcal{A}_{2} z_{t}=\mathcal{N}^{*} g(\mathcal{N} \tilde{w})-\mathcal{N}^{*} g(\mathcal{N} w), \\
& z(0)=z_{t}(0)=0
\end{aligned}
$$

Note that (5.2)-(5.3) has the same form as (3.1)-(3.3) with $h=\mathcal{N}^{*} g(\mathcal{N} \tilde{w})-\mathcal{N}^{*} g(\mathcal{N} w)$ and zero initial conditions. Thus, we can apply the continuous dependence result of Theorem 3.1 to (5.2)-(5.3). In this case it gives that there exists a constant $C>0$ independent of $h$ such that

$$
\|z\|_{\mathcal{Z}} \leq C\|h\|_{L^{2}\left([0, T], \mathcal{V}_{2}^{*}\right)}
$$

(since initial conditions are zero in (5.2)-(5.3)). Now

$$
\|z\|_{\mathcal{V} \times \mathcal{H}}=\left\|\left(\begin{array}{c}
u(T)-\tilde{u}(T) \\
u_{t}(T)-\tilde{u}_{t}(T)
\end{array}\right)\right\|_{\mathcal{V} \times \mathcal{H}} \leq\left\|\left(\begin{array}{c}
u(T)-\tilde{u}(T) \\
u_{t}(T)-\tilde{u}_{t}(T)
\end{array}\right)\right\|_{\mathcal{Z}}=\|z\|_{\mathcal{Z}},
$$

where $\mathcal{Z}$ is defined in (3.7). So to conclude that $\Psi$ is continuous it is enough to show that $\|h\|_{L^{2}\left([0, T], \mathcal{V}_{2}^{*}\right)}$ can be made arbitrarily small by choosing $\|w-\tilde{w}\|_{C\left([0, T], \mathcal{V}_{2}\right)}$ small enough. We have:

$$
\begin{aligned}
& \|h\|_{L^{2}\left([0, T], \mathcal{V}_{2}^{*}\right)}=\left\|\mathcal{N}^{*} g(\mathcal{N} \tilde{w})-\mathcal{N}^{*} g(\mathcal{N} w)\right\|_{L^{2}\left([0, T], \mathcal{V}_{2}^{*}\right)}^{2}=\int_{0}^{T}\left\|\mathcal{N}^{*}(g(\mathcal{N} \tilde{w})-g(\mathcal{N} w))\right\|_{\mathcal{V}_{2}^{*}}^{2} d t \\
& \leq k^{\prime 2} \int_{0}^{T}\|g(\mathcal{N} \tilde{w})-g(\mathcal{N} w)\|^{2} d t
\end{aligned}
$$

Since $g$ is continuous from $\mathcal{H} \rightarrow \mathcal{H}$ and

$$
\|\mathcal{N} \tilde{w}(t)-\mathcal{N} w(t)\|=\|\mathcal{N}(\tilde{w}(t)-w(t))\| \leq \sqrt{\tilde{k}}\|\tilde{w}(t)-w(t)\| \mathcal{V}_{2} \leq \sqrt{\tilde{k}}\|\tilde{w}-w\|_{C\left([0, T], \mathcal{V}_{2}\right)},
$$


we get that by choosing $\|\tilde{w}-w\|_{C\left([0, T], \mathcal{V}_{2}\right)}$ small enough, the right side of (5.4) (and thereby $\|h\|_{L^{2}\left([0, T], \mathcal{V}_{2}^{*}\right)}$ also) can be made as small as required. This proves that $\Psi$ is continuous.

Now note that $\Psi(L)=U_{T}(B)$, so to complete the proof of Lemma 5.1 it is enough to prove that the set $L$ is precompact in $C\left([0, T], \mathcal{V}_{2}\right)$. Using the same version of the Ascoli-Arzela Theorem as in [BGS2, NS], we show that

1) $\{w(t) \mid w \in L\}$ is precompact in $\mathcal{V}_{2}$ for each $t \in[0, T]$,

2) $L$ is equicontinuous.

Statement 1) follows from the fact that $\{w(t) \mid w \in L\}$ is bounded in $\mathcal{V}$ and $\mathcal{V} \hookrightarrow \mathcal{V}_{2}$ is compact (this is the only place where this assumption is used). To show statement 2) we note that:

$$
\begin{aligned}
& \|w(t+\Delta t)-w(t)\|_{\mathcal{V}_{2}}^{2}=\left\|\int_{t}^{t+\Delta t} w_{\tau}(\tau) d \tau\right\|_{\mathcal{V}_{2}}^{2} \\
& \leq\left(\int_{t}^{t+\Delta t}\left\|w_{\tau}(\tau)\right\|_{\mathcal{V}_{2}} d \tau\right)^{2} \leq \Delta t \int_{t}^{t+\Delta t}\left\|w_{\tau}(\tau)\right\|_{\mathcal{V}_{2}}^{2} d \tau \\
& \leq \Delta t \frac{C}{k_{2}}
\end{aligned}
$$

by the apriori estimate in [BGS2]. This completes the proof of Lemma 5.1.

In order to complete the proof of asymptotic compactness the last thing to show is the decay property of $W_{t}$. Since $v$ is the weak solution of (3.18)-(3.20) we can proceed formally and multiply by $v_{t}$ to obtain:

$$
\frac{d}{d t} \frac{1}{2}\left\|v_{t}\right\|^{2}+\frac{d}{d t} \frac{1}{2} \sigma_{1}(v, v)+\operatorname{Re} \sigma_{2}\left(v_{t}, v_{t}\right)=0
$$

Multiplying by 2 and using A5) we get

$$
\frac{d}{d t}\left\|v_{t}\right\|^{2}+\frac{d}{d t} \sigma_{1}(v, v)+2 \frac{k_{2}}{\tilde{c}}\left\|v_{t}\right\|^{2} \leq 2 \lambda_{0}\left\|v_{t}\right\|^{2} .
$$

Let $l=2 \frac{k_{2}}{\tilde{c}}$. Multiplying by $e^{l t}$ we obtain:

$$
e^{l t} \frac{d}{d t}\left\|v_{t}\right\|^{2}+e^{l t} \frac{d}{d t} \sigma_{1}(v, v)+l e^{l t}\left\|v_{t}\right\|^{2} \leq 2 \lambda_{0} e^{l t}\left\|v_{t}\right\|^{2} .
$$

Integrating from 0 to $t$ we obtain

$$
e^{l t}\left\|v_{t}(t)\right\|^{2}-\left\|v_{t}(0)\right\|^{2}+\int_{0}^{t} e^{l s} \frac{d}{d s} \sigma_{1}(v, v) d s \leq 2 \lambda_{0} \int_{0}^{t} e^{l s}\left\|v_{s}(s)\right\|^{2} d s
$$


which after an integration by parts yields:

$$
\begin{aligned}
& e^{l t}\left\|v_{t}(t)\right\|^{2}-\left\|\varphi_{1}\right\|^{2}+e^{l t} \sigma_{1}(v(t), v(t))-\sigma_{1}(v(0), v(0))-\int_{0}^{t} l e^{l s} \sigma_{1}(v, v) d s \\
& \leq 2 \lambda_{0} \int_{0}^{t} e^{l s}\left\|v_{s}(s)\right\|^{2} d s .
\end{aligned}
$$

Using condition A2), A3) and multiplying by $e^{-l t}$ we get:

$$
\left\|v_{t}(t)\right\|^{2}+k_{1}\|v(t)\|_{\mathcal{V}}^{2} \leq e^{-l t}\left(\left\|\varphi_{1}\right\|^{2}+c_{1}\left\|\varphi_{0}\right\|_{\mathcal{V}}^{2}\right)+l_{1} \int_{0}^{t} e^{l(s-t)}\left(\|v(s)\|_{\mathcal{V}}^{2}+\left\|v_{s}(s)\right\|^{2}\right) d s
$$

where $l_{1}=\max \left(l c_{1}, 2 \lambda_{0}\right)$. Let $m=\min \left(1, k_{1}\right)$. Using Gronwall's inequality we have:

$$
\begin{aligned}
& \left\|v_{t}(t)\right\|^{2}+\|v(t)\|_{\mathcal{V}}^{2} \leq \frac{1}{m} e^{-l t}\left(\left\|\varphi_{1}\right\|^{2}+c_{1}\left\|\varphi_{0}\right\|_{\mathcal{V}}^{2}\right) \\
& +\frac{1}{m} \int_{0}^{t} l_{1} e^{l(s-t)} e^{-l s}\left(\left\|\varphi_{1}\right\|^{2}+c_{1}\left\|\varphi_{0}\right\|_{\mathcal{V}}^{2}\right) e^{\int_{s}^{t} \frac{l_{1}}{m} e^{l(\theta-t)} d \theta} d s \\
& \leq \frac{1}{m} e^{-l t}\left(\left\|\varphi_{1}\right\|^{2}+c_{1}\left\|\varphi_{0}\right\|_{\mathcal{V}}^{2}\right)+\frac{l_{1}}{m}\left(\left\|\varphi_{1}\right\|^{2}+c_{1}\left\|\varphi_{0}\right\|_{\mathcal{V}}^{2}\right) \int_{0}^{t} e^{-l t} e^{\frac{l_{1}}{l m}} d s \\
& \leq\left(\left\|\varphi_{1}\right\|^{2}+c_{1}\left\|\varphi_{0}\right\|_{\mathcal{V}}^{2}\right) e^{-l t}\left[\frac{1}{m}+\frac{l_{1}}{m} t e^{\frac{l_{1}}{l m}}\right]
\end{aligned}
$$

Now the right side of this inequality clearly goes to 0 as $t \rightarrow \infty$ so we have the necessary decay. Thus we conclude that the dynamical system is bounded, point-dissipative and asymptotically compact, consequently it has a compact, invariant connected global attractor $\mathcal{A} \subset \mathcal{V} \times \mathcal{H}$

\section{Regularity of the Attractor}

Our next step is to describe the structure of the attractor. We have the following theorem:

Theorem 6.1 Let $\mathcal{E}$ denote the set of fixed points of the dynamical system $\left\{V_{t}, t \geq 0, \mathcal{V} \times \mathcal{H}\right\}$ with the assumption that the real part of the form $\sigma_{2}$ is strictly coercive $\left(\lambda_{0}=0\right.$ in A5). Then the attractor

$$
\mathcal{A}=\mathcal{M}_{+}(\mathcal{E})
$$

where the unstable set $\mathcal{M}_{+}(\mathcal{E})$ of $\mathcal{E}$ is the set

$$
\left.\left\{u_{*} \in \mathcal{V} \times \mathcal{H} \text { and } d\left(V_{t} u_{*}\right), \mathcal{E}\right) \stackrel{t \rightarrow-\infty}{\longrightarrow} 0\right\} .
$$


Proof: The crucial step in the proof is that this dynamical system possesses a Lyapunov function defined on the global attractor. (Actually, in this case the Lyapunov function is global.) Once this is shown, the statement follows by a well-known theorem [Te] p.401. By definition, a function $F$ is a global Lyapunov function for the semigroup $\left\{S_{t}, t \geq 0, X\right\}$ if $F: X \rightarrow \mathbb{R}$ is a continuous function such that

1. for any $\Phi=\left(\begin{array}{c}\varphi_{0} \\ \varphi_{1}\end{array}\right) \in X$ the function $t \rightarrow F\left(S_{t}(\Phi)\right)$ is nonincreasing,

2. if $F\left(S_{\tau} \Phi\right)=F(\Phi)$ for some $\tau$, then $\Phi$ is a fixed point of the semigroup, i.e. $S_{t}(\Phi)=\Phi$ for all $t \geq 0$.

We claim that the function $F: \mathcal{V} \times \mathcal{H} \rightarrow \mathbb{R}$

$$
F(u, v)=\frac{1}{2}\|v\|^{2}+\frac{1}{2} \sigma_{1}(u, u)+G(\mathcal{N} u)-\operatorname{Re}\langle f, u\rangle_{\mathcal{V}_{2}^{*}, \mathcal{V}_{2}}
$$

is a Lyapunov function for the semigroup $\left\{V_{t}, t \geq 0, \mathcal{V} \times \mathcal{H}\right\}$. Statement 1 . is clearly true, since

$$
\begin{aligned}
& \frac{d}{d t} F\left(w, w_{t}\right)=\frac{d}{d t}\left\{\frac{1}{2}\left\|w_{t}\right\|^{2}+\frac{1}{2} \sigma_{1}(w, w)+G(\mathcal{N} w)-\operatorname{Re}\langle f, w\rangle_{\mathcal{V}_{2}^{*}, \mathcal{V}_{2}}\right\} \\
& \leq-\operatorname{Re} \sigma_{2}\left(w_{t}, w_{t}\right) \leq-k_{2}\left\|w_{t}\right\|_{\mathcal{V}_{2}}^{2} \leq 0
\end{aligned}
$$

which shows that $F$ is nonincreasing. Statement 2. follows from the fact that if $F\left(V_{\tau} \Phi\right)=$ $F(\Phi)$, then $\left\|w_{t}(t)\right\|_{\mathcal{V}_{2}}=0$ for a.e. $t \in[0, \tau]$. Since $w_{t} \in C([0, T], \mathcal{H})$ it follows that $w_{t}(t)=0$ for all $t \in[0, \tau]$. Then $w$ must be constant on any time interval (from the semigroup property), so $\left(\begin{array}{c}\varphi_{0} \\ \varphi_{1}\end{array}\right)$ is a fixed point of $V_{t}$.

Continuity of $F$ can be easily verified, so the attractor of the dynamical system is the unstable set of the set of its fixed points.

\section{$7 \quad$ Examples}

Example 7.1 We consider an m-dimensional, nonlinear damped membrane with fixed boundary.

Let $\Omega \subset \mathbb{R}^{m}$ be a bounded domain with $C^{1}$-smooth boundary $\Gamma$. We consider the problem: 


$$
\begin{aligned}
& w_{t t}+\kappa_{1} \Delta^{2} w+\kappa_{2}\left(\Delta^{2}\right)^{\alpha} w_{t}+\mathcal{N}^{*} g(\mathcal{N} w)=f \\
& \left.w\right|_{\Gamma=0} \\
& \left.\frac{\partial w}{\partial n}\right|_{\Gamma}=0 \\
& w(\cdot, 0)=\varphi_{0} \in H_{0}^{2}(\Omega), \quad w_{t}(\cdot, 0)=\varphi_{1} \in L^{2}(\Omega) \\
& x=\left(x_{1}, \cdots, x_{m}\right) \in \Omega, t \in[0, T], \\
& (x, t) \in \Omega \times[0, T] \equiv Q_{T} \\
& 0<\alpha<1 .
\end{aligned}
$$

In this problem $\mathcal{A}_{1}$ is the biharmonic operator, $\Delta^{2}$, where $\Delta=\sum_{j=1}^{m} \frac{\partial^{2}}{\partial x_{j}^{2}}$ is the Laplacian, and $\mathcal{A}_{2}=\left(\Delta^{2}\right)^{\alpha}$ is a fractional power of the biharmonic operator.

$$
\mathcal{V}=H_{0}^{2}(\Omega)=\left\{\psi \in H^{2}(\Omega):\left.\psi\right|_{\Gamma}=\left.\frac{\partial \psi}{\partial n}\right|_{\Gamma}=0\right\}, \mathcal{H}=L^{2}(\Omega)
$$

and

$$
\mathcal{V}_{2}=H_{0}^{2 \alpha}(\Omega)
$$

is a fractional Sobolev space which due to the well-known embedding theorems (see, e.g. [Wl]) can be described explicitly by:

$$
H_{0}^{2 \alpha}(\Omega)= \begin{cases}H^{2 \alpha}(\Omega) & \text { if } 0<\alpha<\frac{1}{4} \\ \left\{\Psi \in H^{2 \alpha}(\Omega):\left.\Psi\right|_{\Gamma}=0\right\} & \text { if } \frac{1}{4} \leq \alpha<\frac{3}{4} \\ \left\{\Psi \in H^{2 \alpha}(\Omega):\left.\Psi\right|_{\Gamma}=\left.\frac{\partial \Psi}{\partial n}\right|_{\Gamma}=0\right\} & \text { if } \frac{3}{4} \leq \alpha<1\end{cases}
$$

In this example $\mathcal{N} \in \mathcal{L}\left(\mathcal{V}_{2}, \mathcal{H}\right)$ can be any bounded linear operator mapping $\mathcal{V}_{2}=H_{0}^{2 \alpha}(\Omega)$ to $\mathcal{H}=L^{2}(\Omega)$, e.g. if $\alpha \geq 1 / 2$ then we can choose $\mathcal{N}=\frac{d}{d x}$ for $m=1$, or for $m>1$ we can let $\mathcal{N}=\Delta^{\beta}$ for any $0<\beta \leq \alpha<1$. We also assume that $f \in \mathcal{V}_{2}^{*}$ and the nonlinear term $g$ is a scalar function $g: \mathbb{R} \rightarrow \mathbb{R}$ such that

$$
G(\xi)=\int_{0}^{\xi} g(\tau) d \tau, \quad g(\xi)=G^{\prime}(\xi)
$$

satisfies the following conditions:

1. There exist positive constants $C_{j}$ for $j=1,2,3$ and $\epsilon>0$ such that

$$
-\frac{1}{2}\left(\kappa_{1}+\kappa_{2}-\epsilon\right)|\xi|^{2}-C_{1} \leq G(\xi) \leq C_{2}|\xi|^{2}+C_{3}
$$


2. There are positive constants $\widetilde{C}_{j}, j=1,2$ such that

$$
|g(\xi)| \leq \widetilde{C}_{1}|\xi|+\widetilde{C}_{2}
$$

3. We assume that

$$
g^{\prime}(\xi) \geq-k_{1}
$$

It is easy to see that such a gatisfies A8)-A11) and all the other conditions (A1)-A7) and A12)) are also satisfied. Thus this system possesses a compact global attractor by Theorem 2.1.

Example 7.2 Let us consider transverse vibrations of a fixed end nonlinear beam of length $\ell$ with structural (or square-root, $\mathcal{A}_{1}^{\frac{1}{2}}$ ) damping [Ru, BIW]:

$$
\begin{aligned}
& w_{t t}+w_{x x x x}-w_{t x x}+g(w)=f \\
& w(t, 0)=w(t, \ell)=w_{x}(t, 0)=w_{x}(t, \ell)=0 \\
& w(0, \cdot)=\varphi_{0} \in H_{0}^{2}(0, \ell) \\
& w_{t}(0, \cdot)=\varphi_{1} \in L^{2}(0, \ell)
\end{aligned}
$$

Now

$$
\begin{aligned}
& \mathcal{V}=H_{0}^{2}(0, \ell)=\left\{\phi \in H^{2}(0, \ell): \phi(0)=\phi^{\prime}(0)=\phi(\ell)=\phi^{\prime}(\ell)=0\right\} \\
& \mathcal{V}_{2}=H_{0}^{1}(0, \ell)=\left\{\phi \in H^{1}(0, \ell): \phi(0)=\phi(\ell)=0\right\}
\end{aligned}
$$

and

$$
\begin{aligned}
& \left\langle\mathcal{A}_{1} \varphi, \psi\right\rangle_{\mathcal{V}^{*}, \mathcal{V}}=\left\langle\varphi_{x x}, \psi_{x x}\right\rangle \\
& \left\langle\mathcal{A}_{2} \varphi, \psi\right\rangle_{\mathcal{V}_{2}^{*}, \mathcal{V}_{2}}=\left\langle\varphi_{x}, \psi_{x}\right\rangle \\
& \mathcal{N}=I .
\end{aligned}
$$

Let $g$ satisfy conditions (7.5)-(7.8), and $f \in L^{2}\left(0, T ; \mathcal{V}^{*}\right)$. Here assumptions A1)-A12) are all satisfied, so by Theorem 2.1 the system possesses a compact global attractor. 
Instead of the structural damping a spatial hysteresis damping [BIW] could also be used in the above example. In that case $\mathcal{V}_{2}=H^{1}(0, \ell)$ and the damping sesquilinear form can be given as

$$
\begin{aligned}
& \left\langle\mathcal{A}_{2} \varphi, \psi\right\rangle=\left\langle(\nu I-G) \varphi_{x}, \psi_{x}\right\rangle, \text { where } \\
& G(\varphi)(x)=\int_{0}^{\ell} \gamma(x, y) \varphi(y) d y \text { and } \\
& \nu(x)=\int_{0}^{\ell} \gamma(x, y) d y .
\end{aligned}
$$

$\gamma$ is a symmetric, non-negative kernel in $L^{\infty}((0, \ell) \times(0, \ell))$. Again if $g$ satisfies conditions (7.5)-(7.8) and $f \in L^{2}\left(0, T ; \mathcal{V}^{*}\right)$ then by Theorem 2.1 the system has a compact global attractor.

Example 7.3 Let $\Omega$ be a smooth bounded domain in $\mathbb{R}^{m}$. Consider the strongly damped nonlinear wave equation

$$
\begin{aligned}
& w_{t t}-\alpha \Delta w_{t}-\Delta w+g(w)=f \\
& w(x, 0)=\varphi_{0}(x) \in H_{0}^{1}(\Omega), \quad w_{t}(x, 0)=\varphi_{1}(x) \in L^{2}(\Omega) \\
& w(x, t)=0, \quad x \in \partial \Omega \\
& x=\left(x_{1}, \cdots, x_{m}\right) \in \Omega, \quad t \in[0, T] \\
& (x, t) \in \Omega \times[0, T] \equiv Q_{T}, \quad \alpha>0
\end{aligned}
$$

In this problem

$$
\mathcal{V}=\mathcal{V}_{2}=H_{0}^{1}(\Omega)
$$

and

$$
\mathcal{A}_{1}=\mathcal{A}_{2}=-\Delta, \mathcal{N}=I .
$$

We assume that $g$ satisfies (7.5)-(7.8), and $f \in \mathcal{V}^{*}$.

We remark that although $\mathcal{V}$ is not compactly embedded in $\mathcal{V}_{2}$ in this case, the existence of a compact global attractor is still guaranteed. If we examine the arguments where this assumption was used, i.e., Lemma 5.1., we can see that they are still valid, since $\mathcal{N}$ in this case is continuous from $\mathcal{H}$ to $\mathcal{H}$. This results in the continuity of $\Psi$ in Lemma 5.1 from $L$ with $w \in C([0, T], \mathcal{H})$ to $\mathcal{V} \times \mathcal{H}$ and then the following arguments are valid since $\mathcal{V}$ is compactly embedded in $\mathcal{H}$. 


\section{Acknowledgment}

I am very grateful to Prof. D. S. Gilliam and Prof. V. I. Shubov for their valuable suggestions, continuous support and encouragement during the preparation of this paper.

This research was supported in part by the US Air Force Office of Scientific Research under grant AFOSR F49620-95-1-0236.

\section{References}

[B] F. Browder, Nonlinear monotone operators and convex sets in Banach spaces, Bull. A.M.S. 71 (1965), 780-785.

[Ba1] J. Ball, Stability theory for an extensible beam, J. Diff. Eqns. 14 (1973), 399-418.

[Ba2] J. Ball, On the Asymptotic Behavior of Generalized Processes, with Applications to Nonlinear Evolution Equations, J. Diff.Eqns 27 (1978), 224-265.

[BGS1] H. T. Banks, D. S. Gilliam, V. I. Shubov, Well-posedness for a one dimensional nonlinear beam, CRSC-TR94-18 (October, 1994), "Computation and Control, IV (Bozeman, MT, 1994)", Birkhäuser Boston, Boston, MA, 1995.

[BGS2] H.T.Banks, D.S. Gilliam, V.I. Shubov, Global Solvability for Damped Nonlinear Hyperbolic Systems, CRSC-TR95-25 (August, 1995), Differential and Integral Equations 10 (1997), 309-332.

[BIW] H. T. Banks, K. Ito, and Y. Wang, Well-posedness for damped second order systems with unbounded input operators, CRSC-TR93-10 (June 1993), Differential and Integral Equations 8 (1995), 587-606.

[CT] S. Chen, R. Triggiani, Proof of extensions of two conjectures on structural damping for elastic systems: the case $\frac{1}{2} \leq \alpha \leq 1$, Pacific J. Math. 39 (1989), 15-55.

[Fa] E. Fašangovà, Attractor for a Beam Equation with Weak Damping, Applicable Analysis 59 (1995), 1-13.

[Da] C.M. Dafermos, Asymptotic behavior of solutions of evolution equations, in "Nonlinear Evolution Equations," Academic Press, (1978).

[H] J. K. Hale, "Asymptotic Behavior of Dissipative Systems," American Mathematical Society, Providence, 1988. 
[Ha] J. K. Hale, N. Stavrakakis, Compact Attractors for Weak Dynamical Systems, Applicable Analysis 26 (1988), 271-287.

[La] O.A. Ladyzhenskaya, "Attractors for Semigroups and Evolution Equations," Cambridge University Press, Cambridge, 1991.

[LSU] O.A. Ladyzhenskaya, V.A. Solonnikov, N.N. Ural'ceva, "Linear and Quasilinear Equations of Parabolic Type," Translations of AMS, Vol. 23, 1968.

[Li] J.L. Lions, "Optimal Control of Systems Governed by Partial Differential Equations," Springer-Verlag, New York, 1971.

[M] G. Minty, Monotone nonlinear operators in Hilbert spaces, Duke Math J., 29 (1962), 341-346.

[NS] A.W. Naylor and G.R. Sell, "Linear Operator Theory in Engineering and Science," Springer Verlag, New York, 1982.

[P] G.A. Pinter, Weak Attractor for Damped Abstract Nonlinear Hyperbolic Systems, (to appear in J. Math Sys. Est. Control).

[Ru] D.L. Russell, On mathematical models for the elastic beam with frequency proportional damping, in "Control and Estimation of Distributed Parameter Systems," SIAM, Philadelphia, 1992.

[S1] M. Slemrod, Asymptotic Behavior of a Class of Abstract Dynamical Systems, $J$. Diff. Eqns. 7 (1970), 584-600.

[Te] R. Temam, "Infinite-Dimensional Dynamical Systems in Mechanics and Physics," Springer Verlag, New York, 1988.

[Wl] J. Wloka, "Partial Differential Equations," Cambridge Univ. Press, 1992. 